\title{
An update on the relevance of vaccine research for the treatment of metastatic melanoma
}

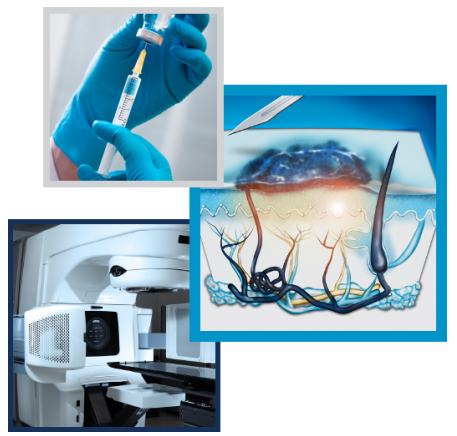

\author{
Robert O Dillman*,1 \\ ${ }^{1}$ Chief Medical Officer, AIVITA Biomedical, Inc; Clinical Professor of Medicine, University of California Irvine, Irvine, CA 92612, USA \\ * Author for correspondence: Tel.: +1 949735 7229; bob@aivitabiomedical.com
}

\section{Practice points}

\section{Past \& present}

- In the past decade, the introduction of effective signal transduction inhibitors and anticheckpoint antibodies has significantly improved the prognosis for patients with metastatic melanoma, but the majority of such patients still die within 5 years.

- Vaccine approaches designed to induce immunity to well-characterized melanoma-associated antigens, or to antigens expressed on allogeneic tumor cell lines, have not resulted in approved agents.

- Despite the limitations associated with the immunosuppressive tumor microenvironment, there now is one intralesional autologous vaccine that has received regulatory approval for patients who have primarily soft-tissue metastases.

Future

- There are still unmet needs that may be met by therapeutic vaccines.

- Successful vaccines likely will be those that induce or enhance recognition of patient-specific autologous neoantigens that result from nonsynonymous mutations contained in autologous tumor cells.

Signal transduction inhibitors and anticheckpoint antibodies have significantly improved survival for metastatic melanoma patients, but most still die within 5 years. Vaccine approaches to induce immunity to well-characterized melanoma-associated antigens, or to antigens expressed on allogeneic tumor cell lines, have not resulted in approved agents. Despite the limitations associated with the immunosuppressive tumor microenvironment, there now is one intralesional autologous vaccine approved for patients who have primarily soft-tissue metastases. There is continued interest in patient-specific vaccines, especially dendritic cell vaccines that utilize ex vivo loading of autologous antigen, thus bypassing certain in vivo immunosuppressive cells and cytokines. Because of their mechanism of action and limited toxicity, they are potentially synergistic or additive to other antimelanoma therapies.

First draft submitted: 31 August 2017; Accepted for publication: 3 November 2017; Published online: 23 November 2017

Keywords: autologous tumor-associated antigens $\bullet$ metastatic melanoma • therapeutic vaccines

A recent commentary concluded that there is a potential role for therapeutic vaccines in cancer therapy despite the clinical success of immune checkpoint inhibitors [1]. A similar conclusion previously was made for metastatic melanoma, in which it was argued that there is a strong rationale for therapeutic anticancer vaccines against metastatic melanoma even though to date no antimelanoma vaccines had been proven to be effective, and the best chance for success rested on approaches leading to recognition of autologous tumor cell antigens (tumor-associated antigen [TAA]) [2]. Furthermore, the suggestion that therapeutic vaccine research was no longer relevant because of the success of new targeted therapies including BRAF/MEK and immune-checkpoint inhibitors was refuted. The following is an update regarding these issues. 


\section{Our current understanding of tumor immunology \& immunotherapy?}

The past 15 years have witnessed dramatic changes in our understanding of tumor immunology and potential immunotherapeutics. We now appreciate that the clinical diagnosis of cancer is the result of an evolutionary process in which certain cancer cells have emerged and persist despite intrinsic mechanisms (such as p53) that can eliminate dysregulated cells, and despite a combination of innate and adaptive immunity that eliminates most cancer cells or maintains an equilibrium that keeps cancer at an undetectable level $[3,4]$.

In terms of the adaptive immune system, it is useful to think of cancer immunotherapy in the context of a tumor immunology continuum that includes antigen processing and immune activation, trafficking of immune cells into tumors via the bloodstream and the interaction of immune cells with tumor cells [5,6]. TAAs are released from dying tumor cells, and the number of TAAs may be increased by radiation, cytotoxic chemotherapy, injected lytic virus or injection of inflammatory cytokines. TAAs are processed by antigen-presenting cells (APC) of which the best known is the dendritic cell (DC), a cell that is at the nexus of innate and adaptive immunity in that it performs phagocytosis as do other macrophages, but it also processes and presents 9-25 amino acid digested peptides in the context major histocompatibility (MHC) complexes to $\mathrm{T}$ lymphocytes via dendrite extensions. The interaction in lymph nodes between TAA presented in the context of MHC molecules by DC and T-cell receptors of T lymphocytes is what triggers the immune response. Presentation of TAA in the context of MHC class I molecules triggers a type 1 helper T-cell response that leads to cytotoxic T lymphocytes (CTLs). This is the process of antigen processing and immune activation and this is where cancer vaccines have their putative role. Activated $\mathrm{T}$ cells must then traffic to the site of tumor where they recognize tumor cells via interaction between the T-cell receptors on T lymphocytes and TAA in the presence of MHC molecules on the tumor cells. While linked to the tumor cells, the CTLs release enzymes such as perforin and granzyme-B, which damage the tumor cells and lead to cell death. Presentation of TAA in the context of MHC class II molecules triggers a type 2 helper T-cell response that leads to B-cell production of antigen-specific monoclonal antibodies that can interact with effector cells of various cytotoxic cells to induce antibody-dependent cell-mediated cytotoxicity.

Surviving cancer cells have evolved to evade the intracellular and innate and adaptive immune mechanisms of cancer control [3,4]. Some of the ways in which this occurs include secretion of ligands that bind to natural killer cells, loss of major MHC antigens, mutations in APC, a microenvironment that includes immunosuppressive cells, such as myeloid-derived suppressor cells (MDSC), regulatory $\mathrm{T}$ cells and macrophages presenting antigen in a manner that induces tolerance rather than immunization. Some of the recognized immunosuppressive cytokines include TGF- $\beta$, IL-10, and indoleamine 2,3 dioxygenase. In addition, there is immune checkpoint inhibition at the level of interaction between APCs and T lymphocytes (CTLA-4) and between T lymphocytes and tumor cells (PD-1/PDL1). At this time, most of the focus on cancer immunotherapy is on altering the agonist-antagonist balance between CTL and tumor cells, and/or cytokines and cells that create a tumor immunosuppressive microenvironment.

A therapeutic vaccine would act at the level of TAA recognition and immune activation. The process can be further divided into three parts: TAA exposure, TAA processing by DC and finally the initiation of an adaptive immune response directed against TAA, based on interaction between DC and T lymphocytes in the lymph nodes. Because vaccines are inducing or enhancing an immune response that may be suppressed, to optimize efficacy, vaccines need to be administered with other immune-enhancing agents.

There have been many failed attempts to create therapeutic anticancer vaccines for melanoma patients [7]. The most important characteristic of a vaccine is its TAAs that act as immunogens that can induce or enhance TAAspecific immunity that could lead to death of cancer cells. The simplest anticancer vaccines are those containing only one or a few well-characterized TAA. Trials of these products have been useful to prove that cancer patients indeed can make immune responses to specific antigens that have been injected with the intent of inducing such responses. However, significant clinical benefit is uncommon, and this approach has yet to succeed in randomized trials testing single or combinations of such characterized antigens. A variation of this shared TAA approach is the use of allogeneic cell lines as the TAA source, but such products also have been unsuccessful in randomized trials. Given interpatient heterogeneity, it should not be surprising that 'one-size-fits-all' vaccines using well-characterized common TAA or allogeneic tumor cells as TAA sources have not succeeded when tested in large numbers of cancer patients. For this reason, many researchers have concluded that the ideal source of TAA is a patient's own cancer cells so that one can exploit the entire repertoire of TAA in that patient. 


\begin{tabular}{|c|c|c|c|c|}
\hline Treatment arm & Number of patients & Response rate (\%) & PFS (months) & OS (months) \\
\hline IL-2 + gp100 & 91 & 16 & 2.2 & 17.8 \\
\hline IL-2 alone & 94 & 6 & 1.6 & 11.1 \\
\hline $\mathrm{p}$-value & & 0.03 & 0.008 & 0.06 \\
\hline
\end{tabular}

OS: Overall survival; PFS: Progression-free survival.

Data taken from [9].

\begin{tabular}{|c|c|c|c|c|c|}
\hline Treat arm & Number of patients & Response rate (\%) & Median PFS (months) & Median OS (months) & 2-year OS (\%) \\
\hline Ipi + gp100 & 403 & 5.7 & 2.8 & 10.0 & 30.0 \\
\hline Ipi alone & 137 & 11.0 & 2.9 & 10.1 & 33.2 \\
\hline gp100 & 136 & 1.5 & 2.8 & 6.4 & 16.3 \\
\hline
\end{tabular}

Ipi: Ipilimumab anti-CTLA-4; OS: Overall survival; PFS: Progression-free survival.

Data taken from [10].

\section{Therapeutic vaccine strategies that have been ineffective in metastatic melanoma}

Well-characterized melanoma tumor-associated antigens

Historically, the development of cancer vaccines was based on empirical observations related to the efficacy of preventive vaccines against pathogens including viruses and various microbes, all of which express proteins that are foreign, and therefore immunogenic to human immune systems. In these instances the antigens are pathogen specific. For this reason, many researchers have sought to identify specific TAA that might be expressed on all or a large subset of patients with melanoma. However, most of the proteins on an individual's cancer cells are self rather than foreign, and therefore are not recognized as antigens by the immune system. In this sense, there are no, or very rare tumor-specific TAA that might serve as effective immunogens for large numbers of patients. In hindsight, such approaches seem overly simplistic and overly optimistic because of the biological heterogeneity of melanoma cells among melanoma patients.

Many of the trials testing well-characterized melanoma TAA were summarized previously $[7,8]$. Until recently, most therapeutic vaccine approaches focused on injection of TAA in combination with some sort of inflammatory agent such as Bacille Calmette-Guérin (BCG) or GM-CSF. In terms of the antigen recognition and immune activation step of tumor immunology, such vaccines were designed to increase exposure to TAA and/or stimulate the processing of TAA by DCs. Trials with such vaccines have consistently demonstrated induction of immune responses in some patients, but rarely clinical benefit. A few of the largest and most recent randomized trials are discussed here.

The one positive randomized trial metastatic melanoma was limited to patients healthy enough to be treated with high-dose IL-2 [9]. For that trial, it took HLA testing of 479 patients to enroll 185 patients for an open-label, randomized trial. Enrollees included 174 patients with stage IV, eight with locally advanced stage III and three had unknown stage. Eligible patients were positive for HLA*A0201 expression and medically suitable for high-dose IL-2; so the study population is not that representative of the general population of metastatic melanoma patients. Gp100 was administered in the adjuvant Montanide ISA-51. The results of the trial are summarized in Table 1.

In a much larger randomized trial, gp100 peptide failed to provide a survival advantage in patients with stage IV $(\mathrm{n}=666)$ and unresectable stage III $(\mathrm{n}=10)$ metastatic melanoma [10]. More than two-thirds had M1c disease, which is more representative of the metastatic melanoma patient population. This large double-blind, randomized trial that led to the approval of monoclonal anti-CTLA antibody ipilimumab was actually designed with a 3:1:1 randomization (ipilimumab + gp100 vaccine vs ipilimumab alone vs gp100 vaccine alone), because it was believed that the most effective therapy would be ipilimumab + gp 100 vaccine. However, all of the benefits demonstrated in this trial were the result of therapy with ipilimumab (Table 2).

A vaccine consisting of a combination of well-characterized HLA-restricted tumor-associated peptides failed to provide a survival advantage [11]. ECOG enrolled 4697 patients in a double-blind, placebo-controlled trial after complete resection of regionally advanced melanoma (stage III, 60\%) or distant metastatic disease (stage IV, 40\%). Because of the requirement to be free of disease at the time of randomization, most of the stage IV patients had M1a or M1b disease (cutaneous and/or lung metastases with a normal lactate dehydrogenase [LDH]) rather than 


\begin{tabular}{|c|c|c|c|c|c|c|}
\hline Vaccine & GM-CSF & Number of patients & Median RFS (months) & 5-year RFS (\%) & Median OS (months) & 5-year OS (\%) \\
\hline Peptides & GM-CSF & 109 & 12.8 & 32.4 & 72.1 & 56.5 \\
\hline Peptides & Placebo & 111 & 10.0 & 33.2 & 63.7 & 51.9 \\
\hline Placebo & GM-CSF & 109 & 11.0 & 30.6 & 69.3 & 51.2 \\
\hline Placebo & Placebo & 107 & 9.8 & 28.0 & 62.2 & 51.6 \\
\hline
\end{tabular}

OS: Overall survival; RFS: Relapse-free survival.

Data taken from [11].

Table 4. Randomized trial of dendritic cell vaccine with peptide antigens versus dacarbazine in patients with metastatic

\section{melanoma.}

\begin{tabular}{|llllll|}
\hline & Number of patients & ORR (\%) & PFS (months) & Median OS (months) & 2-year OS (\%) \\
\hline DC-peptides & 53 & 3.8 & 3.2 & 9.3 & 25 \\
\hline Chemotherapy & 55 & 5.5 & 2.8 & 11.6 & 25 \\
\hline p-value & & & 0.48 \\
\hline $\begin{array}{l}\text { DC-peptide: Dendritic cell loaded with peptide; ORR: Objective response rate; OS: Overall survival; PFS: Progression-free survival. } \\
\text { Data taken from [15]. }\end{array}$
\end{tabular}

M1C disease at the time of resection. As summarized in the table below, the subset of patients positive for HLA-A2 patients $(\mathrm{n}=436)$ had four arms. The vaccine cocktail included three HLA-A2-restricted peptides (tyrosinase, gp100 and MART-1) emulsified in Montanide ISA-51. Patients who were not HLA-2 positive ( $\mathrm{n}=379)$ were randomized between GM-CSF and placebo. GM-CSF was injected subcutaneously at a dose of 250 microgram daily for 2 weeks, then off 2 weeks and repeated every 4 weeks [12]. There was no difference in survival for grouped analysis for all patients based on GM-CSF versus placebo (regardless of peptide vaccine or not), or peptide vaccine or not for HLA-2-positive patients, nor for the individual arms for HLA-2-positive patients (Table 3). The combining of both stage III and stage IV patients makes it difficult to compare these results to trials that enrolled patients by stage.

A GlaxoSmithKline vaccine consisting of MAGE-A3 peptide failed to provide a disease-free survival advantage over placebo in patients with resected metastatic melanoma. Detailed results of the study have still not been published. The failure was announced in brief statement in early September 2013 [13].

Well-characterized melanoma TAA have also been loaded onto DC, as summarized previously [14]. Most of these were small exploratory studies focusing on induction of immune response to the TAA being presented but only rare tumor responses were reported. Pooled data from nine published studies of autologous DC loaded with HLA-2-restricted peptides yielded an objective response rate of only 11/201 (5.5\%) [14]. One randomized trial using this approach was conducted in patients with measurable metastatic melanoma, and it was published over 10 years ago [15]. Autologous DC were loaded with HLA-restricted peptides gp100, tyrosinase, Mage-1 and Mage-3 and injected subcutaneously. The control arm was standard intravenous dacarbazine chemotherapy. The study was designed to test whether the DC vaccine (DCV) approach was superior to chemotherapy. The frequency of severe and life-threatening adverse events was less than $5 \%$ in both arms. There was no difference in various measures of therapeutic benefit (Table 4).

Recent approaches with peptide loading of DC have focused on ways to enhance presentation with MHC class 1 molecules and to enhance DC function. One encouraging series of studies involves TriMixDC-MEL, DC loaded by electroporation with mRNA encoding CD40 ligand, DC70 and TLR4, an HLA class II targeting signal (DCLAMP) and one of four HLA-restricted melanoma antigens (gp100, Mage-1, Mage-3 or tyrosinase) depending on antigen expression in each patient's tumor. The mRNA to be transcribed and translated into proteins may improve antigen processing and presentation by DC. In a Phase II trial 4/15 (27\%) melanoma patients with the desired HLA type had an objective response [16]. In subsequent studies, a 70\% 4-year survival rate was reported for 30 stage III and stage IV patients who were disease free following surgery, then treated with TriMixDC-MEL with or without IFN- $\alpha$ [17]. A 38\% response rate (15/39) was reported in patients with measurable disease in a trial in which the product was combined with anti-CTLA-4 [18]. 


\begin{tabular}{|c|c|c|c|c|}
\hline & Median DFS (months) & 5-Year DFS (\%) & Median OS (months) & 5-year OS (\%) \\
\hline $\begin{array}{l}\text { Allogeneic cell line } \\
\text { vaccine + BCG }\end{array}$ & 42.6 & 47.2 & $>60$ & 59.1 \\
\hline BCG & 47.0 & 52.1 & $>60$ & 67.7 \\
\hline $\begin{array}{l}\text { Allogeneic cell line } \\
\text { vaccine + BCG }\end{array}$ & 8.3 & 27.4 & 31.5 & 39.6 \\
\hline BCG & 7.2 & 20.9 & 38.7 & 44.9 \\
\hline$p$-value & & 0.418 & & 0.245 \\
\hline
\end{tabular}

BCG: Bacille Calmette-Guérin Mycobacterial tuberculosis preparation; DFS: Disease-free survival; OS: Overall survival.

Data taken from [19]

\section{Allogeneic cell lines}

The other shared TAA approach, which also in hindsight would appear to be doomed to failure because of interpatient heterogeneity, is the use of allogeneic cell lines as a source of TAA. Once again the assumption was that malignant melanoma cells from various patients shared many common antigens, even if we had not actually identified them. Another problem with this approach is that many of the proteins from another individual's cells will be considered foreign to the host immune system. Thus, there could be a substantial immune response to a variety of proteins expressed on an allogeneic tumor cell line, but few of those immune responses would actually be directed against the patient's melanoma TAA. It is possible that immunization with such irrelevant antigens might divert existing endogenous antitumor responses. Results from several trials are consistent with this hypothesis.

Several large trials including five large randomized trials that enrolled 217 to 1160 patients, all failed to demonstrate a survival relative benefit for the allogeneic TAA approach in patients after resection of stage IIB-IV melanoma $[7,8]$. The most recent and largest of these randomized studies utilized three allogeneic cell lines that were selected because of their expression of numerous well-characterized melanoma TAA. The irradiated cells were admixed with BCG and injected subcutaneously. Randomized double-blind trials with a BCG alone control arm were conducted in 1160 patients with surgically resected stage III disease, and a similar trial enrolled 496 patients with stage IV melanoma who had no evidence of disease after resection of metastatic lesions [19]. At the time of randomization, all patients had remained disease free during the 6 weeks following surgery. The survival data from those two trials are summarized in Table 5 and provide useful reference points for survival rates in the modern era. Unfortunately, at the time of closure all trends favored the control arm, which was actually an active immunotherapy arm rather than a placebo. As might be expected, survival was much better for patients who had stage III rather than stage IV disease at the time of complete resection of all metastases.

TAA from allogeneic cell lines have been loaded onto autologous DC in a number of small studies. Data pooled for three studies that used autologous DC loaded with TAA from allogeneic tumor cell lines had a response rate of $6 / 68(8.8 \%)$ [14]. This approach has not been tested in randomized trials.

\section{Current status of autologous therapeutic vaccine approaches for metastatic melanoma}

There are many reasons why autologous cancer cells are the best source of TAA for any given patient [8]. Evidence supporting this view has steadily increased during the past decade. For instance, in addition to the disappointing clinical results with peptide and allogeneic cancer cell line vaccines, we now know that the vast majority of metastatic melanoma cells are associated with hundreds to thousands of nonsynonymous mutations that may result in transcription and translation into proteins that are foreign to that patient's immune system, that is, they are neoantigens, and therefore immunogenic [20-24]. Most of the nonsynonymous mutations in cancer cells are passenger rather than driver mutations. Even though many common driver genes are mutated, the specific mutations and the specific epitopes of the translated proteins differ from patient to patient. Signal transduction pathways associated with such mutations are the target of enzyme inhibitors, but the antigen-specific mutations associated with these tend to be patient specific, as are the passenger mutations. 


\begin{tabular}{|c|c|c|c|c|c|c|}
\hline Treatment arm & Number of patients & Durable ORR (\%) & ORR (\%) & Median TTF (months) & Median OS (months) & 3-year OS (\%) \\
\hline Intralesional Tvec & 295 & 16 & 26 & 8.2 & 23.2 & 39 \\
\hline $\begin{array}{l}\text { Subcutaneous } \\
\text { GM-CSF }\end{array}$ & 141 & 2 & 6 & 2.9 & 18.9 & 30 \\
\hline$p$-value & & $<0.001$ & $<0.001$ & & 0.051 & \\
\hline
\end{tabular}

It is now possible to analyze the entire mutanome to identify all of the possible antigenic neoantigens and generate vaccines targeting such epitopes. Melanoma patients immunized with DC loaded with TAA selected by exomic analysis and mRNA transcription exhibited immune responses to previously unrecognized TAA, and showed increased immune reactivity to previously recognized TAA [25]. These observations lead to the conclusion that the best source of TAA for each patient is his/her own tumor, or more specifically, own tumor cells. Toward this end, there now a number of approaches being tested that utilize whole tumor, mRNA from whole tumor and short-term autologous tumor cell lines, rather than going through the tedious process of trying to identify all relevant neoantigens. Several trials using these approaches were summarized previously [7-8,14].

\section{Intralesional injections as a therapeutic vaccine}

The easiest way to take advantage of autologous TAA in patients with detectable metastases is with intralesional injections of agents that either increase the availability of TAA for processing by DC and/or enhance the function of DC in antigen processing and/or antigen presentation to $\mathrm{T}$ lymphocytes. The 1898 dramatic reports of cancer regression following injection of William B Coley's famous 'toxins' derived from bacterial cell cultures of Streptococcus and Serratia were based on intralesional injections, even though by 1915, he had ceased to administer the concoctions intralesionally [26]. A theoretical disadvantage of the intralesional approach is the immunosuppressive microenvironment within a tumor lesion because the processing and loading of DC with TAA in the tumor microenvironment may be limited because of the local immunosuppressive effects from cytokines, regulatory $\mathrm{T}$ cells, macrophages and myeloid-derived suppressor cells.

There is now one commercially available therapeutic vaccine that takes into account the importance of autologous TAA, and that is talimogene laherparepvec (Tvec), which consists of oncolytic Herpes simplex virus transfected to encode genes resulting in secretion of GM-CSF. This product is injected into tumor nodules where autologous tumor cells can be lysed by the virus, and where secretion of GM-CSF and the presence of other inflammatory cytokines can promote differentiation of monocyte-macrophages into DC that process the released TAA and then traffic to lymph nodes to induce an immune response or stimulate memory $\mathrm{T}$ cells that recognize specific antigen. The lysis itself can result in regression of tumor size, and the immunization component could result in tumor effects at distant noninjected lesions.

Tvec was approved based on a higher durable response rate (6 months) in an open-label, 2:1 randomized trial that compared intralesional Tvec with GM-CSF [27]. The design of this trial is subject to criticism because instead of being injected intralesionally as part of a double-blind design, GM-CSF in the control arm was injected subcutaneously at a dose of $125 \mu \mathrm{g} / \mathrm{m}^{2}$ weekly for 2 weeks, then off 2 weeks and repeated every 4 weeks [12]. As noted earlier in this review, in a double-blind, randomized trial, this same GM-CSF regimen failed to show an advantage compared with placebo in metastatic melanoma patients rendered free of disease by surgery [11]. The results are summarized in the Table 6.

The patient population for this trial is not representative of most metastatic melanoma trials because of the selection of patients who had cutaneous nodules or lymph nodes that could be easily injected, such that most patients had in transit or regional disease, and only a few patients had other than cutaneous distant metastases. In terms of a putative therapeutic vaccine effect, unfortunately there were only a few responses in noninjected lesions. However, subsequent studies suggest that the combination of intralesional injections of Tvec with ipilimumab are associated with a response rate that appears to be much higher than reported for either agent alone, but this would require confirmation in a randomized trial [28]. Tvec is also being tested in combination with PD-1 inhibitors.

In contrast to the clinical trial success of Tvec was the failure of intralesional velimogene aliplasmid (Allovectin-7). There is a strong rationale for optimizing the TAA processing by DCs for presentation in the context of MHC class 
Table 7. Randomized trial of intralesional vaccine to optimize dendritic cell processing and presentation of

tumor-associated antigen versus intravenous dacarbazine in patients with advanced melanoma.

Study arm

Number of patients

ORR (\%)

OS (months)

4-year OS (\%)

Allovectin

260

4.6

18.6

25

Dacarbazine

130

12.3

24.1

25

$\mathrm{p}$-value

0.491

ORR: Objective response rate; OS: Overall survival.

Data taken from [29].

1 antigens [14]. Rather than increasing exposure to TAA, the rationale for the Allovectin product was enhancement of DC processing of available TAA for presentation to $\mathrm{T}$ cells. The product consisted of a plasmid lipid complex containing DNA-encoding $\beta 2$-microglobulin and the HLA-B7 histocompatibility molecule that is common in individuals of northern and western European descent. Once ingested by APC, the DNA can be transcribed and translated to produce the two subunits to reconstitute MHC class 1 for presentation of antigen by DC to type 1 helper T cells that drive CTL responses. Basically, an allogeneic MHC class 1 molecule was being manufactured intracellularly in the hopes that available antigen would more likely be processed in a manner leading to CTL. HLA typing was not an eligibility criteria for the clinical trial in which intralesional Allovectin was compared with standard cytotoxic chemotherapy with a primary end point of objective response rate of all lesions per RECIST. In a 2:1 randomized trial in 390 patients distributed roughly a third stage III (regional disease), a third stage M1a (cutaneous only and normal LDH) and a third lung as the only site of visceral metastases and normal LDH, the response rate was higher for dacarbazine chemotherapy (Table 7) [29]. The median survival was much longer for those in the chemotherapy arm, but there was no difference in overall survival and no difference in the proportion surviving 4 or 5 years. Among the few objective responders, survival was better for those treated with Allovectin (p $=0.018$ ) and the proportions alive at 4.5 years were $6 / 12$ in the Allovectin arm and only $1 / 16$ in the chemotherapy arm. Thus, the subset analysis among responders suggests that Allovectin did produce long-term clinical benefit in a subset of patients. As expected drug-related serious adverse events and drug-related grade 3 or 4 adverse events occurred in a higher proportion of patients treated with chemotherapy.

Building on Coley's reports, the modern interest in intralesional injections as melanoma immunotherapy dates to the late 1960s with BCG. The late Don Morton reported regression of $89 \%$ of 780 lesions injected with BCG in 45 patients and regression of noninjected lesions in $13 \%$ of the patients [30]. In a 59-patient randomized trial, intralesional BCG was associated with a response rate of $45 \%$ compared with only $9 \%$ in patients treated with cutaneous BCG, and the intralesional therapy was associated with more systemic toxicity and a longer median survival [31]. Subsequent efforts focused more on using BCG as an adjuvant for melanoma vaccines rather than as an intralesional therapy.

Various clinically available cytokines, including IFN- $\alpha$ since the mid-1980s, and IL-2, and GM-CSF since the early 1990s, have been injected intralesionally in melanoma patients, but there has been very little systematic reporting of such clinical practice, and no commercial entities have sought marketing approval from regulators for intralesional injection of these agents. However, one group did report a complete response rate of $70 \%$ among 48 stage III melanoma lesions injected intralesionally with $\geq 3$ million international units IL-2 [32].

The studies point out that intralesional injection of a variety of agents have been associated with regression of injected lesions, but regression of noninjected lesions, sometimes referred to as an 'abscopal' effect, and evidence for a vaccine effect are much less common. Recently other products have been injected into melanoma lesions. The rationale for each of these is either to increase the release of TAA and/or enhancement of DC activity. Intralesional rose Bengal (PV-10), which causes lysis of tumor cells, yielded a 51\% ORR in target lesions in an 80-patient trial (78\% stage III disease), but only rare responses in noninjected lesions were noted [33]. L19-IL-2 is recombinant antibody-cytokine fusion protein that consists of IL-2 and one arm of monoclonal antibody L19 that reacts with the extracellular-domain B of fibronectin. Injections of L19-IL-2 resulted in a response rate of $54 \%$ in the injected lesions among 24 stage III melanoma patients [34].

\section{Autologous tumor to generate therapentic vaccines}

Autologous tumor samples collected surgically have been used to produce a variety of patient-specific melanoma vaccines ex vivo using the tumor as a source of TAA. A randomized trial of heat shock proteins derived from 


\begin{tabular}{|c|c|c|c|c|c|}
\hline Treatment & Number of patients & Median PFS (months) & 3-year PFS (\%) & Median OS (months) & 3-year OS (\%) \\
\hline TCV & 74 & 4.5 & 19 & 20.5 & 36 \\
\hline DCV & 54 & - & - & $>60$ & 64 \\
\hline DCV & 24 & 5.4 & 16 & 43.4 & 61 \\
\hline TCV & 18 & 3.7 & 18 & 20.5 & 25 \\
\hline $\mathrm{p}$-value & & 0.50 & & & 0.018 \\
\hline
\end{tabular}

autologous tumor failed to show a benefit compared with a placebo [35]. Others have loaded the TAA derived from autologous tumor into DC by phagocytosis [36], or by loading DC with TAA via electroporation [37], or using electroporation to internalize the mRNA from the tumor into DC where it is translated into autologous TAA $[17,38]$. These approaches have to be tested in randomized trials. There also have been efforts to establish short-term autologous tumor cell cultures to serve as an irradiated tumor cell (ITC) vaccine (TCV) [39], or as a source of TAA for loading onto DC to create autologous DCV [40,41]. As summarized elsewhere, a major challenge with the whole tumor approach is the variability in numbers of cancer cells that are actually derived from a tumor sample, and retention of immunosuppressive cells and cytokines in some procedures [7,8]. Extracting autologous tumor cell mRNA and then introducing it into DC via electroporation is an interesting approach because the mRNA can then produce the TAA intracellularly, which theoretically could increase presentation with MHC class 1 molecules to drive CTL responses. However, electroporation also damages DC and decrease their viability.

Current evidence suggests that antigen presentation via DC is more effective if the antigen source is autologous tumor rather than well-characterized TAA. As reviewed elsewhere, for seven studies utilizing DC + autologous cells or lysate in which ORR was reported, ORR was 14/115 (12.2\%) as opposed to $11 / 201$ (5.5\%) from nine published studies of autologous DC loaded with HLA-2-restricted peptides [14]. Furthermore, survival data have been especially encouraging among patients treated with vaccines that utilized short-term autologous tumor cell lines as the source of patient-specific TAA [42]. A 74-patient trial of injected autologous ITCs from short-term cell lines was associated with a 5 -year survival rate of $28 \%$ [39]. Subsequently, a 54-patient trial of autologous DC loaded with TAA from ITC from a short-term cell line (DCV) was associated with a 5 -year survival rate of $50 \%$ [40]. This was followed by a randomized trial comparing DCV to TCV with both products admixed with GM-CSF. Because of an unplanned premature closure, an early analysis was performed that showed a survival benefit for patients randomized to the DCV arm [41]. Long-term follow-up has confirmed a more than doubling of overall survival despite no difference in progression-free survival. DCV treatment was the only variable associated with survival beyond 3 years in a multivariate analysis, at a time when all patients were dead or followed beyond 3 years with no patients lost to follow-up [43]. To date, this is the only direct comparison of a DCV versus a TCV using autologous cancer-cell-derived TAA.

A Phase III double-blind, randomized, placebo-controlled trial was initiated to test the DCV eltrapuldencel-T in patients with metastatic melanoma [44]. That trial was stopped prematurely by the company that was sponsoring the trial, and no clinical results are available [45].

Despite recent improvement in survival since introduction of checkpoint inhibitors, there is still an unmet need that therapeutic vaccines might meet

There is no question that interest in anticancer vaccines has waned in the face of the failure of several large randomized vaccine trials, and the success of immune checkpoint and signal transduction inhibitors. However, there still appears to be an unmet need that could be filled by therapeutic vaccines [1]. As summarized in Table 8, on the basis of clinical data, it is apparent that the majority of patients with metastatic melanoma are not getting long-term survival benefit from these newer therapies, even though they are clearly superior to previous cytotoxic chemotherapy approaches and an improvement over cytokine therapy with agents such as IL-2 or IFN- $\alpha$. The monoclonal antibody anti-CTLA-4 ipilimumab was approved by the US FDA in 2010, vemurafenib was approved for BRAF-mutated melanoma in 2011, the MEK inhibitor trametinib was approved for BRAF-mutated melanoma in 2013 and two anti-PD-1 monoclonal antibodies (nivolumab and pembrolizumab) were approved in 2014. The combination of BRAF and MEK inhibitors is now preferred because of higher response rates and decreased adverse 
Table 9. Summary of results from trials of signal transduction inhibitors and immune checkpoint inhibitors in patients

with metastatic melanoma.

\begin{tabular}{|llllll|}
\hline Therapy & ORR (\%) & Median PFS (months) & Median OS (months) & 5-year OS (\%) & Grade 3 or 4 AE (\%) \\
\hline BRAF/MEK & 75 & 11 & 25 & 60 \\
\hline Anti-CTLA-4 & 20 & 3 & 20 & 20 \\
\hline Anti-PD-1 & 45 & 7 & 38 & $\sim 35$ \\
\hline CTLA-4/PD-1 & 60 & 12 & $>36$ & 20 \\
\hline
\end{tabular}

Data taken from [46-52].

AE: Adverse events; ORR: Objective response rate; OS: Overall survival; PFS: Progression-free survival.

events, although severe and life-threatening toxicity is still a concern. Examples include dabrafenib plus trametinib, vemurafenib plus cobimetinib and encorafenib and binimetinib. The combination of anti-CTLA- 4 plus anti-PD-1 appears to be an advantage over single agent anti-PD-1 but is associated with significant toxicity. Median and 5-year survival data are emerging from trials of the newer therapies that have received regulatory approval since 2010 . Table 9 provides a general summary of these results, relying mostly on data from randomized trials and recent meeting presentations [46-52]. It is evident from this table that metastatic melanoma is seldom being cured with these newer agents, and that toxicity is still a problem. Therefore an unmet therapeutic need still persists.

A number of other agents that can facilitate checkpoint agonists or block checkpoint antagonists are being tested in combination with the established agents. However, all of these approaches assume that there has already been TAA recognition (CTL-tumor cell interaction), or that TAA recognition is in progress (DC-T-cell interaction). Vaccines can also be combined with these other agents. Of note, in B16 melanoma mouse models, the combination of gp100 vaccine with anti-CTLA-4 and GM-CSF [53], or gp100 vaccine with anti-PD-1 and GM-CSF [54], resulted in better tumor control than either vaccine plus GM-CSF or either checkpoint inhibitor plus GM-CSF.

Gene studies of melanoma samples suggest a more favorable prognosis in association with upregulation of genes associated with an immune response (e.g., ICOS, CD3d, ZAP70, TRAT1, TARP, GZMK, LCK, CD2, CXCL13, CCL19, CCR7 and VCAM1) [55]. Immune signatures based on T-cell infiltration are also prognostic of survival. Now evidence of immune suppression can be derived from biopsies of metastatic cancer specimens. Immune signatures can provide not only prognostic information but also be predictive for responses to certain immunotherapies and sequencing of therapies [56]. There are three general immune signatures classified by histologic analysis of tumor specimens that appear to be prognostic and at least partially predictive of a response to immunotherapy. These three groups include: (1) inflammation: infiltration with activated T cells with elevated PD-1/PDL-1) on tumor cells and/or immune infiltrating cells (40-45\%); (2) peripheral T-cell distribution without infiltration and without elevated PD-1/PD-L1 (15-20\%); and (3) no immune response: no T-cell infiltration and no elevated PD-1/PD-L1 (40-45\%) [57,58]. Theoretically immune stimulating agents such as IL-2 should be effective in the first, immune checkpoint modification is often effective in the second, but new immune responses are needed in the setting in which there is no infiltration of $\mathrm{T}$ lymphocytes. Theoretically therapeutic vaccines may add benefit in all three settings, but the greatest unmet need from an immunotherapy perspective is the third group in which there appears to have been no T-cell activation and infiltration. For group 2, exomic analysis shows a high mutation load, often in association with mutations in DNA repair genes, and is usually associated with response to anti-PD-1 therapy. In Group 3, lack of T-cell infiltration and PD-L1 expression is associated with mutations in genes that regulate mesenchymal transition, cell adhesion, remodeling of the extracellular matrix, angiogenesis and wound healing [59]. The only reason vaccines would have nothing to contribute is if all possible TAA recognition and immune responses have already taken place, and those that are suppressed can be liberated with the right combination of agonist and anti-antagonists related to checkpoint control, to optimize induction of the immune response and retaining the ability to kill tumor cells.

Because there are so many mechanisms for cancer cells to evade an effective immune response, combinations and sequences of immune modifying approaches will be needed to optimize outcomes. Effective vaccines that can increase endogenous immune recognition of TAA should still have a role, and an occasional patient might even benefit from a therapeutic vaccine as an effective monotherapy. Because of their lack of toxicity, patient specificity and potential to target all neoantigens in a given patient, vaccine approaches focused on autologous TAA deserve further research and consideration. 


\section{Summary}

There is a persistent unmet clinical need in the treatment of metastatic melanoma because: the majority of patients do not benefit from anticheckpoint therapy, there is evidence that not all patients make immune responses to their tumors, there is evidence that immune responses to autologous tumor antigens can be induced by patient-specific vaccines and there is clinical evidence from the precheckpoint era that suggests survival can be positively impacted by patient-specific vaccines DCV.

\section{Future perspective}

For the near term, melanoma immunotherapy will continue to build on the success of the immune checkpoint inhibitors by employing various other agonists and antagonists of the immune response in combination with antiPD-1 and anti-PD-L1 antibodies. Some of the newer agents such as anti-indoleamine 2,3 dioxygenase (epacadostat), CD137 and CD137 ligand, OX40 and OX40 ligand and CD40 and CD40 ligand, and inducible co-stimulatory ligand (ICOS) and B7RP1, enhance DC and their interaction with T lymphocytes. All of these combinations assume there are sufficient TAA for immune recognition, and all that is needed is to control and modify the endogenous immune response at the level of antigen processing and communication by DC, and to release suppressed anti-TAA immune responses.

Trials are ongoing that combine intralesional vaccine approaches with the currently approved immune checkpoint inhibitors. The lytic GM-CSF-secreting H. simplex product for intralesional injection (Tvec), which combines increased autologous TAA release with local DC stimulation, currently is the only approved vaccine for such combinations. The vaccine effects of this approach appear to be limited, and it is doubtful that ongoing trials that attempt to increase TAA release by radiation therapy and/or chemotherapy in combination with immune checkpoint inhibitors will yield substantially greater survival benefit for patients. It will take time to sort out the benefits and limitations of various combinations of existing and emerging immune modulators, but it is likely that the issue of optimizing TAA exposure will persist, and therefore interest in vaccine research will continue.

For an autologous vaccine strategy other than intralesional injection, at this time the most encouraging approaches are those with autologous DC loaded ex vivo with TAA derived from autologous cancer cells. The advantage of these approaches is that they bypass the immunosuppressive microenvironment that inhibits DC processing of TAA, and therefore limit the vaccine effects of an intralesional injection. The best source of autologous TAA remains to be seen, but it seems likely that approaches with autologous mRNA and/or short-term cell lines will yield more consistent results than efforts involving whole tumor. Based on the results of DC-vaccine trials, it appears that these have limited therapeutic efficacy against measurable disease as a monotherapy, and the benefits of inducing new anti-TAA immune responses may be enhanced if they are combined with other agents that enhance DC-T-lymphocyte activation and that block suppression at the level of interaction between tumor cells and CTLs.

\section{Financial \& competing interests disclosure}

The author is employed by AIVITA Biomedical, Inc. and has shares of stock in Caladrius Biosciences, Inc. The author has no other relevant affiliations or financial involvement with any organization or entity with a financial interest in or financial conflict with the subject matter or materials discussed in the manuscript apart from those disclosed.

No writing assistance was utilized in the production of this manuscript.

\section{Open access}

This work is licensed under the Attribution-NonCommercial-NoDerivatives 4.0 Unported License. To view a copy of this license, visit http://creativecommons.org/licenses/by-nc-nd/4.0/.

\section{References}

Papers of special note have been highlighted as: $\bullet$ of interest; $\bullet \bullet$ of considerable interest

1. Dillman RO. Is there a role for therapeutic cancer vaccines in the age of checkpoint inhibitors? Hum. Vaccin. Imunother. 13(3), 528-532 (2017).

2. Dillman RO. Is vaccine research still relevant for metastatic melanoma? Melanoma Manag. 1(2), 91-94 (2014).

3. Schreiber RD, Old LJ, Smyth MJ. Cancer immunoediting: integrating immunity's roles in cancer suppression and promotion. Science 331(6024), 1565-1570 (2011).

4. Mittal D, Gubin MM, Schreiber RD, Smyth MJ. New insights into cancer immunoediting and its three component phases - elimination, equilibrium and escape. Curr. Opin. Immunol. 27, 16-25 (2014). 
5. Chen DS, Mellman I. Oncology meets immunology: the cancer-immunity cycle. Immunity 39(1), 1-10 (2013).

6. Chen DS, Mellman I. Elements of cancer immunity and the cancer-immune set point. Nature 541(7637), 321-330 (2017).

-• Excellent recent review of the various components of cancer immunity, cancer resistance and opportunities for immunotherapy.

7. Dillman RO. Melanoma vaccines: trials and tribulations. Vaccine 3, 57-78 (2013).

8. Dillman RO, Cornforth AN, Nistor G. Cancer stem cell antigen-based vaccines: the preferred strategy for active specific immunotherapy of metastatic melanoma? Expert Opin. Biol. Ther. 13(5), 643-656 (2013).

9. Schwartzentruber DJ, Lawson DH, Richards JM et al. Gp100 peptide vaccine and interleukin-2 in patients with advanced melanoma. N. Engl. J. Med. 364(22), 2119-2127 (2011).

10. Hodi FS, O'Day SJ, McDermott DF et al. Improved survival with ipilimumab in patients with metastatic melanoma. N. Engl. J. Med. 363(8), 711-723 (2010).

11. Lawson DH, Lee S, Zhao F et al. Randomized, placebo-controlled, Phase III trial of yeast-derived granulocyte-macrophage colony-stimulating factor (GM-CSF) versus peptide vaccination versus GM-CSF plus peptide vaccination versus placebo in patients with no evidence of disease after complete surgical resection of locally advanced and/or stage IV melanoma: a trial of the Eastern Cooperative Oncology Group-American College of Radiology Imaging Network Cancer Research Group (E4697). J. Clin. Oncol. 33(34), 4066-4076 (2015).

12. Spitler LE, Grossbard ML, Ernstoff MS et al. Adjuvant therapy of stage III and IV malignant melanoma using granulocyte-macrophage colony-stimulating factor. J. Clin. Oncol. 18(8), 1614-1621 (2000).

13. GlaxoSmithKline press release. The investigational MAGE-A\# antigen-specific cancer immunotherapeutic does not meet first co-primary endpoint in Phase III melanoma clinical trial. (2013). www.gsk.com/en-gb/media/press-releases/the-investigational-mage-a3-antigenspecific-cancer-immunotherapeutic-does-not-meet-first-co-primary-endpoint-in-phase-iii-melanoma-clinical-trial/

14. Dillman RO, Nistor G, Cornforth AN. Dendritic cell vaccines for melanoma: past, present and future. Melanoma Manag. 3(4), 267-283 (2016).

- Recent review focusing on status of dendritic cell (DC) vaccines in melanoma.

15. Schadendorf D, Ugurel S, Schuler-Thurner B et al. Dacarbazine (DTIC) versus vaccination with autologous peptide-pulsed dendritic cells (DC) in first-line treatment of patients with metastatic melanoma: a randomized Phase III trial of the DC study group of the DeCOG. Ann. Oncol. 17(4), 563-570 (2006).

16. Van Lint S, Wilgenhof S, Heirman C et al. Optimized dendritic cell-based immunotherapy for melanoma: the TriMix-formula. Cancer Immunol. Immunother. 63(9), 959-967 (2014).

17. Wilgenhof S, Corthals J, Van Nuffel AM et al. Long-term clinical outcome of melanoma patients treated with messenger RNA-electroporated dendritic cell therapy following complete resection of metastases. Cancer Immunol. Immunother. 64(3), 381-388 (2015).

18. Wilgenhof S, Corthals J, Heirman C et al. Phase II study of autologous monocyte-derived mRNA electroporated dendritics cells (TRiMixDC-MEL) plus ipilimumab in patients with pretreated advanced melanoma. J. Clin. Oncol. 34(12), 1330-1338 (2016).

19. Morton DL, Mozzillo N, Thompson JF et al. An international, randomized, Phase III trial of bacillus Calmette-Guerin (BCG) plus allogeneic melanoma vaccine (MCV) or placebo after complete resection of melanoma metastatic to regional or distant sites. Presented at: 43rd Annual Meeting of the American Society for Clinical Oncology. IL, USA, 1-5 June 2007 (Abstract 8508).

20. Lennerz V, Fatho M, Gentilini $\mathrm{C}$ et al. The response of autologous $\mathrm{T}$ cells to a human melanoma is dominated by mutated neoantigens. Proc. Natl Acad. Sci. USA 102(44), 16013-16018 (2005).

21. Schumacher TN, Schreiber RD. Neoantigens in cancer immunotherapy. Science 348(6230), 69-74 (2015).

22. Ward JP, Gubin MM, Schreiber RD. The role of neoantigens in naturally occurring and therapeutically induced immune responses to cancer. Adv. Immunol. 130, 25-74 (2016).

23. Vormehr M, Diken M, Boegel S et al. Mutanome directed cancer immunotherapy. Curr. Opin. Immunol. 39(4), 14-22 (2016).

24. Bobisse S, Foukas PG, Coukos G et al. Neoantigen-based cancer immunotherapy. Ann. Transl. Med. 4(14), 262 (2016).

25. Carreno BM, Magrini V, Becker-Hapak M et al. Cancer immunotherapy. A dendritic cell vaccine increases the breadth and diversity of melanoma neoantigen-specific T cells. Science 348(6236), 803-808 (2015).

-• Important proof-of-principle study in which exomic analyses mRNA was used to identify unique autologous neoantigens that were loaded onto autologous DCs, and induced new immune responses and enhanced weak existing immune responses.

26. Nauts HC, Swift WE, Coley BL. The treatment of malignant tumors by bacterial toxins as developed by the late William B. Coley, M.D., reviewed in the light of modern research. Cancer Res. 6, 205-2016 (1946).

- Classic paper discussing the specifics of 'Coley's toxins' which have driven the field of cancer immunotherapy for more than a century, and which may be interpreted as the first intralesional autologous vaccine.

27. Andtbacka RH, Kaufman HL, Collichio F et al. Talimogene laherparepvec improves durable response rate in patients with advanced melanoma. J. Clin. Oncol. 33(25), 2780-2788 (2015).

- Randomized Phase III trial that led to approval of intralesional autologous vaccine approach. 
28. Puzanov I, Milherm MM, Minor D et al. Talimogene laherparepvec in combination with ipilimumab in previously untreated, unresectable stage IIb-IV melanoma. J. Clin. Oncol. 34(22), 2619-2626 (2016).

29. Clinical Trials Database: NCT00395070. https://clinicaltrials.gov/ct2/show/NCT00395070

30. Morton D, Eilber F, Holmes E et al. BCG immunotherapy of malignant melanoma: summary of a seven-year experience. Ann. Surg. 180(4), 635-643 (1974).

31. Nathanson L, Schoenfield D, Regelson W et al. Prospective comparison of intralesional and multipuncture BCG in recurrent intradermal melanoma. Cancer 43(5), 1630-1635 (1979).

32. Weide B, Derhovanessian E, Pflugfelder A et al. High response rate after intratumoral treatment with interleukin-2. Results from a Phase II study in 51 patients with metastasized melanoma. Cancer 116(17), 4139-4146 (2010).

33. Thompson JF, Agarwala SS, Smithers BM et al. Phase II study of intralesional PV-10 in refractory metastatic melanoma. Ann. Surg. Oncol. 22(7), 2135-2142 (2015).

34. Weide B, Eigentler TK, Pflugfelder A et al. Intralesional treatment of stage III metastatic melanoma patients with L19-IL2 results in sustained clinical and systemic immunologic responses. Cancer Immunol. Res. 2(7), 668-678 (2014).

35. Testori A, Richards J, Whitman E et al. Phase III comparison of vitespen, an autologous tumor-derived heat shock protein gp 96 peptide complex vaccine, with physician's choice of treatment for stage IV melanoma: the C-100-21 Study Group. J. Clin. Oncol. 26(6), 955-962 (2008).

36. De Rosa F, Ridolfi L, Flammenghi L et al. Dendritic cell vaccination for metastatic melanoma: a 14 year monoinstitutional experience. Melanoma Res. 27(4), 351-357 (2017).

37. Wolfraim LA, Takahara M, Viley AM et al. Clinical scale electroloading of mature dendritic cells with melanoma whole tumor cell lysate is superior to conventional lysate co-incubation in triggering robust in vitro expansion of functional antigen-specific CTL. Int. Immunopharmacol. 15(3), 488-497 (2013).

38. Kyte JA, Aamdal S, Dueland S et al. Immune response and long-term clinical outcome in advanced melanoma patients vaccinated with tumor-mRNA-transfected dendritic cells. Oncoimmunology 5(11), e1232237 (2016).

39. Dillman RO, DePriest C, DeLeon C et al. Patient-specific vaccines derived from autologous tumor cell lines as active specific immunotherapy: results of exploratory Phase I/II trials in patients with metastatic melanoma. Cancer Biother. Radiopharm. 22(3), 309-321 (2007).

40. Dillman RO, Selvan SR, Schiltz PM et al. Phase II trial of dendritic cells loaded with antigens from self-renewing, proliferating autologous tumor cells as patient-specific anti-tumor vaccines in patients with metastatic melanoma: final report. Cancer Biother. Radiopharm. 24(3), 311-319 (2009).

41. Dillman RO, Cornforth AN, Depriest C et al. Tumor stem cell antigens as consolidative active specific immunotherapy: a randomized Phase II trial of dendritic cells versus tumor cells in patients with metastatic melanoma. J. Immunother. 35(8), 641-649 (2012).

-• Randomized trial demonstrating superior survival for patients treated with autologous DC vaccines loaded with autologous tumor antigens ex vivo compared with autologous irradiated tumor cells that would depend on in vivo antigen processing by DCs.

42. Dillman RO, Selvan SR, Schiltz PM. Patient-specific dendritic-cell vaccines for metastatic melanoma. N. Engl. J. Med. 355(11), 1179-1181 (2006).

43. Dillman RO, McClay EF, Amatruda TT et al. Randomized trial of dendritic vs tumor cell patient-specific vaccines: 5-year analysis. Presented at: 107th Annual Meeting of the American Association for Cancer Research. LA, USA, 15 April 2016.

44. Javed A, Sato S, Sato T. Autologous melanoma cell vaccine using monocyte-derived dendritic cells (NBS20/eltrapuldencel-T). Future Oncol. 12(6), 751-762 (2016).

45. Caladrius press release: Caladrius tightens strategic focus and provides 2016 revenue guidance. (2016). www.caladrius.com/press-release/strategic-focus-and-provides-2016-revenue-guidance/

46. Robert C, Karaszewska B, Schachter J et al. Improved overall survival in melanoma with combined dabrafenib and trametinib. N. Engl. J. Med. 372(1), 30-39 (2015).

47. Schadendorf D, Hodi S, Robert C et al. Pooled analysis of long-term survival data from Phase II and Phase III trials of ipilimumab in unresectable or metastatic melanoma. J. Clin. Oncol. 33(17), 1889-1894 (2015).

48. Robert C, Schachter J, Long GV et al. Pembrolizumab versus ipilimumab in advanced melanoma. N. Engl. J. Med. 372(26), 2532-2532 (2015).

49. Larkin J, Chiarion-Sileni V, Gonzalez R et al. Combined nivolumab and ipilimumab or monotherapy in untreated melanoma. N. Engl. J. Med. 373(1), 23-34 (2015).

50. Wolchok JD, Chiarion-Sileni V, Gonzalez R et al. Overall survival with combined nivolumab and ipilimumab in advanced melanoma. N. Engl. J. Med. 377(14), 1345-1356 (2017).

51. Long GV, Weber JS, Infante JR et al. Overall survival and durable responses in patients with BRAF V600-mutant metastatic melanoma receiving dabrafenib combined with trametinib. J. Clin. Oncol. 34(8), 871-878 (2016). 
52. Ugurel S, Röhmel J, Ascierto PA et al. Survival of patients with advanced metastatic melanoma: the impact of novel therapies. Eur. J. Cancer 53(1), 125-134 (2016).

53. van Elsas A, Hurwitz AA, Allison JP. Combination immunotherapy of B16 melanoma using anti-cytotoxic T lymphocyte-associated antigen 4 (CTLA-4) and granulocyte/macrophage colony-stimulating factor (GM-CSF)-producing vaccines induces rejection of subcutaneous and metastatic tumors accompanied by autoimmune depigmentation. J. Exp. Med. 190(3), 355-366 (1999).

54. Li B, VanRoey M, Wang C et al. Anti-programmed death-1 synergies with granulocytes macrophage colony-stimulating factor - secreting tumor cell immunotherapy providing therapeutic benefit to mice with established tumors. Clin. Cancer Res. 15(5), 1623-1634 (2009).

55. Bogunovic D, O’Neill DW, Belitskaya-Levy I et al. Immune profile and mitotic index of metstatic melanoma lesions enhance staging in predicting patient survival. Proc. Natl Acad. Sci. USA 106(48), 20429-20434 (2009).

56. Chen PL, Roh W, Reuben A et al. Analysis of immune signatures in longitudinal tumor samples yields insight into biomarkers of response and mechanisms of resistance to immune checkpoint blockade. Cancer Discov. 6(8), 627-637 (2016).

- Recent paper defining immune signatures as prognostic markers for survival and predictive markers of benefit from various immunotherapies.

57. Taube JM, Anders RA, Young GD et al. Colocalization of inflammatory response with B7-h1 expression in human melanocytic lesions supports an adaptive resistance mechanism of immune escape. Sci. Transl. Med. 4(127), 127ra37 (2012).

58. Kluger HM, Zito CR, Barr ML et al. Characterization of PD-L1 expression and associated T cell infiltrates in metastatic melanoma samples from variable anatomic sites. Clin. Cancer. Res. 21(13), 3052-3060 (2015).

59. Hugo W, Zaretsky JM, Sun L et al. Genomic and transcriptomic features of response to anti-PD-1 therapy in metastatic melanoma. Cell 165(1), 35-44 (2016). 
